\title{
Non-Syndromic Unilateral Gingival Enlargement - A Case Report
}

\author{
Dr. Shridevi Adaki, ${ }^{1}$ Dr. Suraj Chougule, ${ }^{2}$ Dr. Raghavendra Adaki ${ }^{3}$ \\ ${ }^{I}$ Associate ProfessorDepartment of Oral Medicine and RadiologyB. V. D. U. Dental College Sangli. \\ ${ }^{2}$ Assistant ProfessorDepartment of PeriodonticsB. V. D. U. Dental College Sangli. \\ ${ }^{3}$ Associate ProfessorDepartment of ProsthodonticsB. V. D. U. Dental College Sangli.
}

\begin{abstract}
An unusual case presented here of a 16 years old female patient who came with chief complaint of severe, unilateral, gingival enlargement confined to the left side of oral cavity. Medical history revealed mild mental retardation and cataract surgery done for both eyes 5 years back. A careful recording of the case history and results of clinical examination and investigations were noted to arrive at the diagnosis.
\end{abstract}

Key Words: mental retardation, gingival hyperplasia, cataract surgery.

\section{Introduction}

Gingiva is part of oral mucosa which surrounds the neck of the teeth like a collar. Healthy gingiva is important for the proper functioning of oral cavity and esthetics. Gingival enlargement or hyperplasia is one of the gingival diseases in which there is increase in the cellular matrix. The overgrowth might be caused by several etiological factors, such as the administration of specific drugs (e.g., cyclosporin, nifedipine, phenytoin), the autosomal-dominant or the autosomal-recessive inheritance as an isolated feature, and/or as a syndromic manifestation. ${ }^{1}$ Gingival hyperplasia causes aesthetic, functional, psychological and masticatory disturbances of the oral cavity.

The enlargement may be associated with one or more teeth, involve one or more quadrant(s), or may be generalized. The lingual or palatal gingivae are typically increased in thickness when compared to the bucccal side. The etiology is thought to be familial or idiopathic. The familial variation may occur as an isolated finding or be associated to one of several hereditary syndromes, e.g., Zimmermann-Laband, Murray-Puretic-Drescher, Rutherfurd, multiple hematomas, and Cross syndrome. The exact cause of this abnormality is unknown, but it is thought to be an autosomal dominant pattern of inheritance in most cases. However, autosomal recessive examples have also been noted. ${ }^{2}$

The present case is unusual because, in-spite of having positive medical history and family history still does not fit under criteria of any syndrome.

\section{Case Report}

A 16 year-old girl presented to the Department of Oral medicine and Radiology with the chief complaint of swelling in the gums around the left upper and lower back teeth region and inability to chew food from left side. History revealed that the swelling present since two years with no associated symptoms and gradually increased to its present size. The patient came for treatment only when the swelling started interfering with mastication. Patient gave history of unilateral mastication. There was family history of consanguineous marriage and medical history revealed bilateral cataract surgery 5 years back and patient was mentally retarded and not on any medication for the same.

General physical examination and extraoral examination was done to check for any facial anomalies and abnormalities in maxillofacial complex, but no abnormality was detected.

On intraoral examination, gingival hyperplasia was seen from mesial of first premolar to distal of second molar on the left side, both in maxillary and mandibular arch (Fig 1,2). The right side of the oral cavity was unaffected. The enlargement seemed to be progressing around the $2^{\text {nd }}$ molars. The enlargement involved the marginal, attached and the interdental gingiva. Both facial and lingual/palatal gingiva was affected. The enlarged gingiva was smooth but firm, exhibited no change in the color /altered surface characteristics and extended on to the occlusal surface of maxillary and mandibular $2^{\text {nd }}$ molars. There was no significant pain for the patient and probing revealed little subgingival calculus. The jaws appeared distorted because of the bulbous enlargement of the gingiva.

Based on the history and clinical examination pubertal unilateral gingival enlargement due to hormonal changes was given as provisional diagnosis as age of initiation correlates with that. Since there was absence of abnormalities in extraoral examination association of syndrome with this condition was excluded.

Routine hematological investigations revealed hemoglobin count of $12.3 \mathrm{gm} \%$ and a differential leukocyte count of polymorphonuclear leukocytes (neutrophils) 58\%, lymphocytes 38\%, monocytes 2\%, 
eosinophils $2 \%$, and a random blood sugar level of $98 \mathrm{mg} / \mathrm{dl}$. Assessment of sex hormones does not reveal any abnormality. Radiographic examination (OPG and IOPA X-rays) revealed no gross bone loss (Fig 3, 4).

Gingivectomy was done and tissue sample was sent for histopathological examination. Histopathological examination showed an increase in the amount of connective tissue that was relatively avascular and consisted of increased fibrocellular content. There were numerous immature collagen fibers with abundant fibroblasts. The surface epithelium was acanthotic and parakeratotic, no giant cells were present. Healing was uneventful; sutures were removed after one week.

Based on the investigatory results, final diagnosis non-syndromic unilateral gingival enlargement was given. Gingivectomy of maxillary and mandibular arches has been done and healing was uneventful (Fig 5). Patient is under periodic follow up and there were no signs of recurrence.

\section{Discussion}

Gingival hyperplasia characterized by slowly progressive, non-hemorrhagic, fibrous enlargement of maxillary and mandibular gingival. Clinically, the onset is consistent with the eruption of permanent dentition. Overgrowth can be observed varying in extent and severity. The excess gingival tissue may cover partial or whole crown, resulting in diastemas, teeth displacement, retention of primary teeth, or impacted teeth. The hyperplastic gingiva is usually normal in color, with firm consistency and heavy stippling. ${ }^{3}$

Gingival hyperplasia can be hereditary condition and investigations are in evolution to establish the genetic linkage associated with it. ${ }^{4}$ The autosomal dominant form is often associated with hypertricosis, corneal dystrophy, nail defects, deafness and craniofacial deformities whereas in the autosomal- recessive form with facial anomalies, hypertelorism have been observed but most forms are without defects, other than gingival enlargement. Consanguinity has been in the recessive form. Clinical abnormalities most commonly with gingival enlargement are hirsutism, epilepsy, oligodontia, mental redardation, nystagmus, strabismus, cataract, soft tissue tumors and enlarged facial bones.

In the present case, initially it appeared to be associated with one of the syndromes. The relevant factors for such assumptions were consanguineous marriage of parents, history of cataract surgery and mental retardation. But on detailed examination and investigations no other abnormalities were detected which can fit into the criteria of the syndrome. The above mentioned findings can only be coincidental findings or can be unidentified syndrome till now. This aspect requires further study to confirm the same. Even the sex hormones were in the normal ranges which excludes the chances of pubertal gingival enlargement. By the above information the reason for the enlargement was not clear but after the Gingivectomy patient is comfortable with no signs of recurrence.

So in cases of gingival enlargement, thorough case history and investigations are mandatory to identify the underlying causes or associated syndromes. In the present case no such findings were noticed.

References

[1]. Poulopoulos A, Kittas D, Sarigelou A. Current concepts on gingival fibromatosis-related syndromes. Journal of Investigative and Clinical Dentistry 2011; 2:156-61.

[2]. Sandhu SP, Kakar V,Gogia G, and Narula SC. Unilateral gingival fibromatosis with localized aggressive periodontitis (involving first molars): An unusual case report. J Indian SocPeriodontol. 2009 May-Aug; 13(2): 109-113.

[3]. Long He and Fei-Yun Ping. Gingival fibromatosis with multiple unusual findings: report of a rare case. International Journal of Oral Science 2012;4:221-5.

[4]. Hart TC, Pallos D, Bozzo L, Almeida OP, Marazita ML, O’Connell JR, et al. Evidence of genetic heterogeneity for hereditary gingival fibromatosis. J Dent Res 2000;79:1758-64.

[5]. Sharma S, Goyal D, Shah G, Ray A. Familial gingival fibromatosis: A rare case report. Contemporary Clinical Dentistry 2012:3;s63-s66.

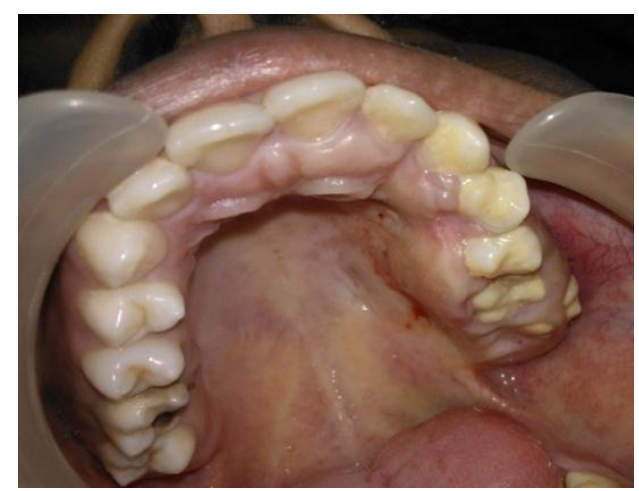

Fig 1: Gingival enlargement extending from mesial aspect of premolar to distal of second molar seen on right side of maxilla involving marginal, interdental and attached gingiva covering the occlusal surface of second molar. 


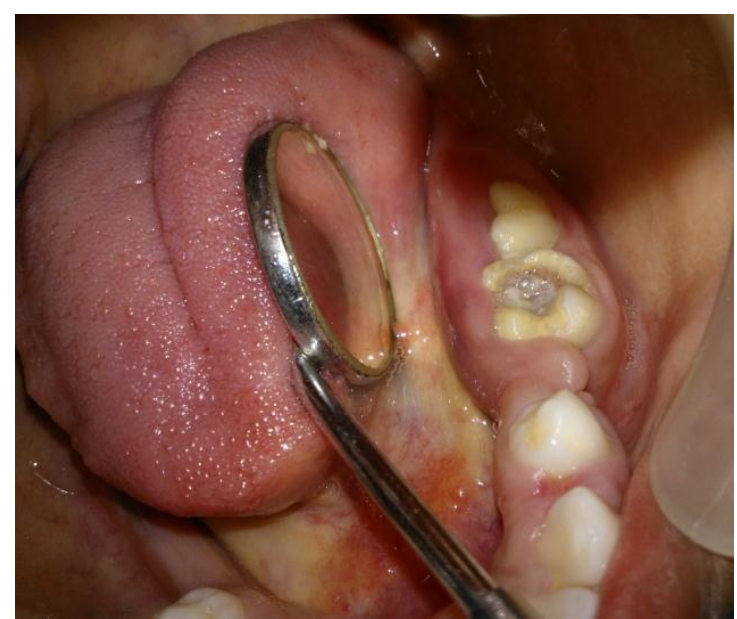

Fig 2: Gingival enlargement seen around left mandibular molars and premolar and covering occusal aspect of second molar.

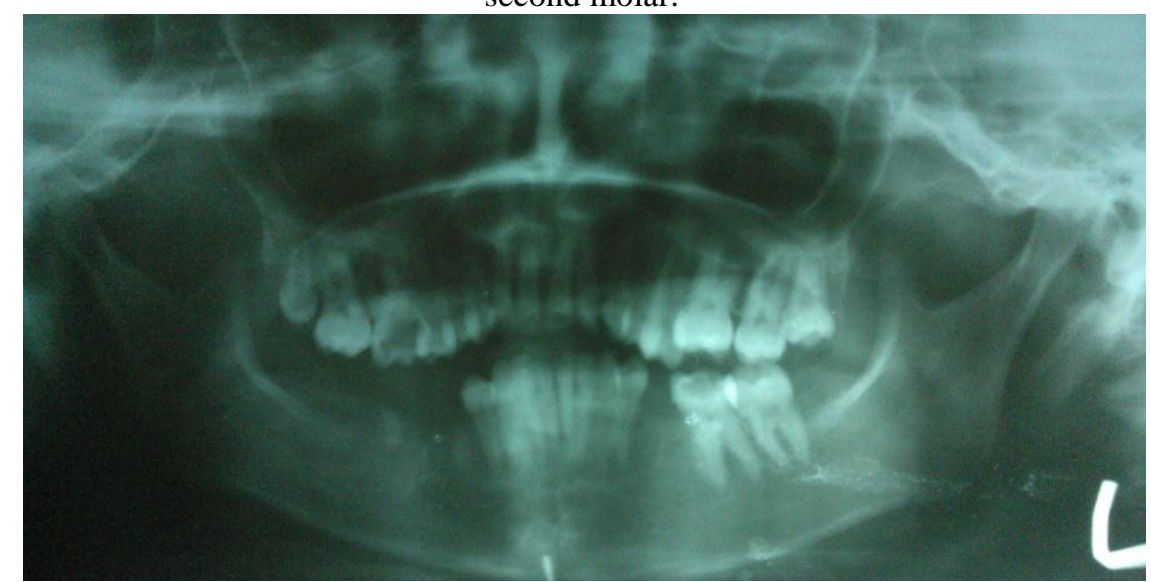

Fig 3: Panoramic radiograph showing no or minimal amount of bone loss.

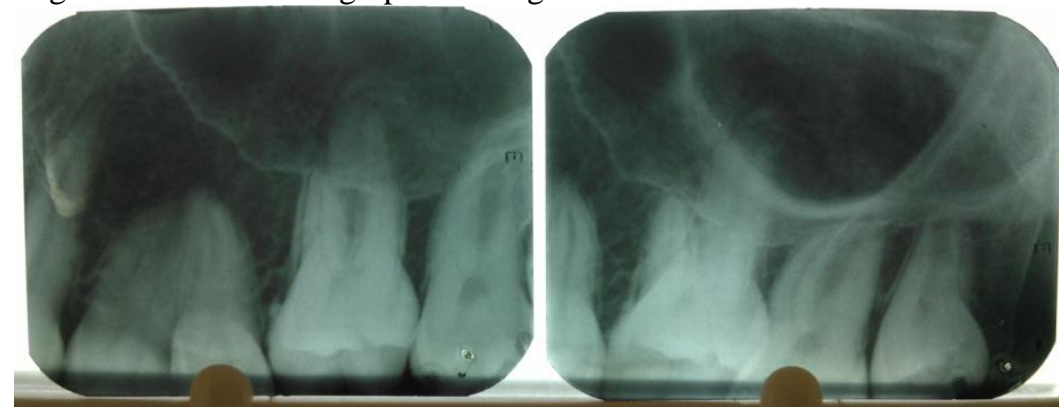

Fig 4: intra-oral radiograph of maxillary premolars and molars showing no bone loss.

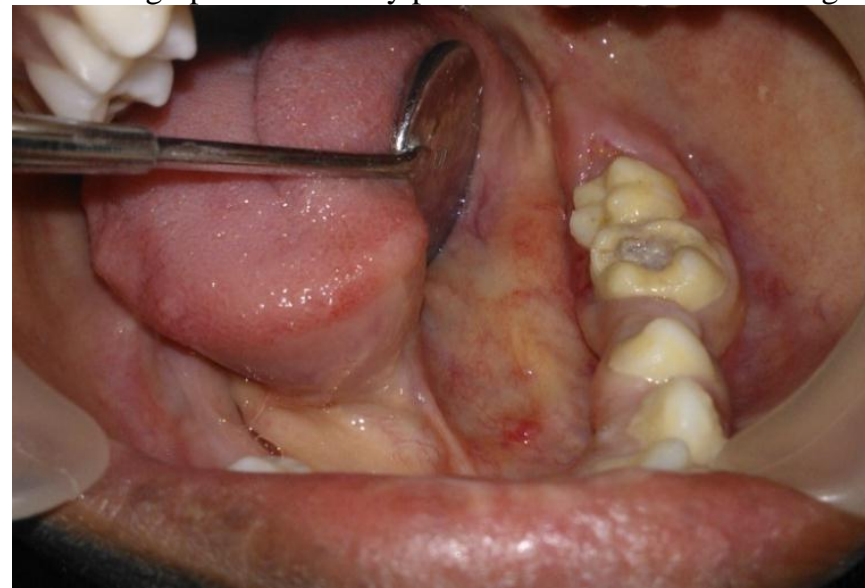

Fig 5: Photograph showingMandibular arch one week after the gingivectomy 